\title{
Urinary $\mathrm{N}$-acetyl- $\beta$-glucosaminidase and eGFR may identify patients to be treated with immuno-suppression at diagnosis in idiopathic membranous nephropathy.
}

Claudio Bazzi*, Tomoko Usui§ Virginia Rizza", Daniela Casellato ${ }^{\circ}$, Masaomi Nangaku§

*D'Amico Foundation for Renal Disease Research.

"Biochemical Laboratory, San Carlo Borromeo Hospital.

${ }^{\circ}$ Nephrology and Dialysis Unit, San Carlo Borromeo Hospital.

Milan, Italy

$\S$ Division of Nephrology and Endocrinology, the University of Tokyo School of Medicine, Tokyo, Japan

Corresponding Author:

Claudio Bazzi, MD

D'Amico Foundation for Renal Disease Research

Via Cherubini, 6; 20145 Milan, Italy

Phone: +39 3388319049

Fax: +3902 48110814

e-mail: claudio.bazzi@alice.it

Running title: Risk stratification by urinary NAG/C and eGFR in IMN

Abstract: 248 words

Text: 2830 words

Abstract 
Background. The clinical course of Idiopathic Membranous Nephropathy (IMN) varies from spontaneous remission of nephrotic syndrome (NS) to end-stage renal disease (ESRD). Selecting patients with high risk of progression for immunosuppressive therapy is mandatory.

Methods. 86 IMN subjects were followed for median of 69 months (range 6-253). Receiver operating characteristic curve and Cox proportional hazard model were used to evaluate prognostic factors for progression, defined as ESRD or eGFR reduction $\geq 50 \%$ of baseline.

Results. Among all, 24 subjects had progression. Area under the ROC curve of $\mathrm{N}$-acetyl- $\beta$-glucosaminidase/creatinine ratio $(\mathrm{NAG} / \mathrm{C})$ were significantly higher than proteinuria/day ( 0.770 and 0.637 respectively, $\mathrm{p}=0.018$ ). In multivariable Cox proportional hazard regression analysis, $\mathrm{NAG} / \mathrm{C}$ and eGFR were independent predictors of progression. Compared to lowest tertile of NAG/C $(<9.4 \mathrm{UI} / \mathrm{gC})$ or highest tertile of eGFR $(\geq 88$ $\left.\mathrm{ml} / \mathrm{min} / 1.73 \mathrm{~m}^{2}\right)$, the hazard ratio of highest tertile of NAG/C $(\geq 19.2)$ was 18.97 (95\%CI, 1.70-211.86) and lowest tertile of eGFR (<59) was 11.58 (95\%CI, 2.02-66.29). Subjects with high NAG/C or low eGFR (high-risk, n.43) had increased progression rate compared to moderate to low NAG/C and high eGFR (low-risk, n.43) with or without NS at baseline (Log-rank test $\mathrm{p}=0.001$ and 0.006 , respectively). In NS subjects $(\mathrm{n}=65)$, in high-risk group progression rate was significantly higher ( $91 \%$ vs. $29 \%, \mathrm{p}=0.003$ ) and remission significantly lower ( $0 \%$ vs. $42 \%, \mathrm{p}<0.001)$ in nonimmunosuppressed compared to steroids and cyclophosphamide treated patients; no significant differences were observed in low-risk group. 
Conclusions. IMN subjects with high NAG/C and low eGFR have increased risk of progression, and immunosuppressive treatment is suggested at diagnosis.

\section{Introduction}

In last decade great advances have been made in knowledge of pathophysiology and treatment of idiopathic membranous nephropathy (IMN). The discovery of podocyte antigen M-type phospholipase A2 receptor $[\mathrm{PLA}(2) \mathrm{R}]$ and its corresponding IgG autoantibodies confirmed the autoimmune nature of IMN (review in 1). Anti-PLA2R antibodies are present in $70-80 \%$ of IMN patients; their serum level correlates with degree of proteinuria and predict outcome, as high values are associated with low probability of spontaneous or treatment-induced remission (2-7). Prediction of progression evaluated in the third tertile of anti-PLA2R antibodies showed that the end point $25 \%$ serum creatinine ( $\mathrm{sCr}$ ) increase or $\mathrm{sCr} \geq 1.30 \mathrm{mg} / \mathrm{dL}$ was $69 \%$ vs. $25 \%$ in the first tertile (8). However at present anti-PLA2R antibodies level do not exactly identify patients who will enter spontaneous remission and candidates to supportive therapy and patients at risk for progression which need immunosuppressive therapy at diagnosis. Recently Rituximab (RTX), the monoclonal antibody directed to podocyte surface antigen CD20 of B cells, responsible of synthesis of anti- PLA(2)R autoantibodies, has been recognized as effective and safe treatment of IMN with nephrotic syndrome (NS) inducing remission in two-thirds of patients often unresponsive to other immunosuppressive agents (9-12). RTX, as more specific and safer treatment in comparison 
with non-specific agents often associated with serious adverse events, has been suggested as first-line therapy for IMN (13). RTX proposal as firstline therapy in IMN with NS, characterized by about 30-40\% of spontaneous remission, requires the availability of baseline risk stratification able to identify patients with high probability of spontaneous remission and long-lasting normal renal function (NRF) which should be treated with supportive therapy and patients with high risk of progression which need immunosuppressive treatment at diagnosis; thus such risk stratification could be of paramount importance for early therapeutic decisions and baseline selection of patients which should be treated or not with IS-therapy. Studies to determine biomarkers value to predict prognosis and treatment responsiveness are still needed (14). Thus the aim of the study was to assess the proteinuric and functional prognostic factors that allow risk stratification with such predictive value in a longitudinal study of 86 IMN patients with rather long follow-up.

\section{Patients}

The study included 86 IMN patients diagnosed between January 1992 and April 2006 at the Nephrology and Dialysis Unit of San Carlo Borromeo Hospital, Milan, Italy. Inclusion criteria: clinical presentation: persistent non-nephrotic proteinuria (PP, $\mathrm{n}=14$ ) or nephrotic syndrome (NS, $\mathrm{n}=72:$ 24-hour proteinuria $\geq 3.5 \mathrm{~g}$ and/or serum albumin $<3.0 \mathrm{~g} / \mathrm{dL}$ ); typical features at light and immunofluorescence microscopy; no clinical, imaging and laboratory signs of secondary $\mathrm{MN}$; at least 6 glomeruli in renal biopsy. The study complied with the Declaration of Helsinki and the 
local requirements for ethical approval. All patients gave informed written consent. Baseline characteristics of patients are reported in Table 1.

\section{Laboratory analysis and renal biopsies}

Proteinuria was measured in 24 hour urine collection and in second morning urine sample by the Coomassie blue method (modified with sodium-dodecyl-sulphate) and expressed as 24 hour proteinuria and protein creatinine/ratio ( $\mathrm{mg}$ urinary protein/g urinary creatinine). Serum $(\mathrm{sCr})$ and urinary creatinine $(\mathrm{uCr})$ were measured enzymatically and expressed in $\mathrm{mg} / \mathrm{dL}$. Serum and urinary IgG, $\alpha 2$-macroglobulin $(\alpha 2 \mathrm{~m})$, albumin, transferrin and $\alpha 1$-microglobulin $(\alpha 1 \mathrm{~m})$ were measured by immunonephelometry as described ; N-acetyl- $\beta$-glucosaminidase (NAG) was measured by colorimetric method as described (16) and expressed as $\mathrm{NAG/creatinine} \mathrm{ratio} \mathrm{(NAG/C).} \mathrm{Glomerular} \mathrm{filtration} \mathrm{rate} \mathrm{(eGFR)} \mathrm{was}$ estimated using the Chronic Kidney Disease Epidemiology Collaboration (CKD-EPI) formula . Renal biopsies were performed and evaluated by previously described standard histological and immunofluorescence methods (18).

\section{Follow up, treatment and outcome}

A rather long follow up was available in all 86 patients (median 69 months; range 6-268). Fourteen patients had PP and 72 NS; the 14 PP patients were treated with supportive therapy; of them 9 had nonnephrotic proteinuria at biopsy that lowered over time; 5 patients had nonnephrotic proteinuria at the first study of proteinuria performed sometime after renal biopsy and a previous history of untreated NS; 30 NS patients were treated with supportive therapy such as diuretics, anti-hypertensives 
(including ACEi [angiotensin converting enzyme inhibitor]/ARB [angiotensin II receptor blockers]), statins, anti-platelet agents, and vitamin D3 when indicated; 35 NS patients, besides supportive therapy, were treated at diagnosis with steroids and cyclophosphamide according to Ponticelli protocol (19); 7 NS patients were treated with steroids alone: prednisolone $1 \mathrm{mg} / \mathrm{kg} /$ day with tapering for $4-12$ months (Fig. 1). The patients not lost to follow up were screened up until the last planned follow-up visit in 2015.

The functional outcome evaluated after a rather long follow-up was very variable: we identified four different outcomes:

1) Clinical Remission [complete (proteinuria $\leq 0.20 \mathrm{~g} / 24$ hours), partial (proteinuria $\leq 2.0 \mathrm{~g} / 24$ hours)] with baseline and last NRF: $\mathrm{n}=39$ ( $\mathrm{n}=9$ with PP and NRF; $\mathrm{n}=30$ with NS and NRF); clinical remission in NS patients with baseline and last stable chronic renal failure (CRF): $n=7$

2) Persistent nephrotic syndrome with baseline and last NRF: $n=8$;

3) Persistent nephrotic syndrome with eGFR reduction from $51 \pm 24$ to $39 \pm 11 \mathrm{ml} / \mathrm{min} / 1.73 \mathrm{~m}^{2}$ at last observation: $\mathrm{n}=8$;

4) Progression: end stage renal disease (ESRD) or eGFR reduction $\geq 50 \%$ : $\mathrm{n}=24$.

\section{Statistical analysis}

Continuous variables are expressed as means \pm SD. Urinary markers were log-transformed before statistical analyses due to their skewed distribution, and expressed as geometric means (95\% confidence 
interval). The renal function decline during the follow-up period was calculated by: eGFR change $=[$ eGFR(last $)-$ eGFR(1st) $] /$ Follow-up $\times 12$ $\left(\mathrm{ml} / \mathrm{min} / 1.73 \mathrm{~m}^{2} /\right.$ year$)$. The change of proteinuria was calculated by: Proteinuria change $=$ Proteinuria $($ last $)-$ Proteinuria $(1 \mathrm{st})(\mathrm{g} / 24 \mathrm{hr})$. The differences of median for continuous values between the study groups were determined using the Wilcoxon rank-sum test. Receiver Operating Characteristic (ROC) curve was plotted to determine the area under the curve (AUC) to compare the predictive ability of proteinuric markers to discriminate between progressors and non-progressors. Cox proportional hazard model was used to assess unadjusted and multivariable-adjusted hazard ratio on the population as a whole. Kaplan-Meier curves and Logrank tests were used to compare progression or remission rate according to risk levels or treatment. Time of progression or remission was calculated from the date of diagnosis. Patients were censored at the time of renal death or at the end of follow-up. All statistical analyses were performed using Stata 12.1 (StataCorp LP, TX, USA). Two-sided $\mathrm{p}<0.05$ was considered statistically significant.

\section{RESULTS}

\section{ROC analysis of proteinuric markers}

Area under ROC curve (AUC) analysis to compare the predictive ability for progression was performed for all eight proteinuric markers (Fig. 2). Compared to proteinuria/24hr, UP/C, Alb/C, $\alpha 1 \mathrm{~m} / \mathrm{C}$, and NAG/C had significantly higher AUC (Table 2). After adjustment with age, sex, serum albumin, blood pressure, and ACEi/ARB treatment, hazard ratio (HR) of baseline eGFR and NAG/C were significant (Table 3). The multivariable- 
adjusted HR for increase of eGFR was 0.96 (95\% CI: 0.94, 0.98), and every 10-time increment of NAG/C was 20.98 (95\% CI: 2.70, 162.69). Thus baseline eGFR and NAG/C were both independent markers for risk of progression.

\section{Functional outcome (progression, remission) according to}

\section{tertiles of NAG/C in all 86 patients.}

The progression rate in lowest (NAG/C $<9.4, \mathrm{n}=28$ ), middle $(9.4 \leq \mathrm{NAG} / \mathrm{C}<19.2, \mathrm{n}=29)$, and highest tertile of NAG/C $(\mathrm{NAG} / \mathrm{C} \leq 19.2$, $\mathrm{n}=29$ ) were $4 \%, 28 \%$ and $52 \%$, respectively (Figure 3 ). The difference was significant between lowest and highest tertile $(p<0.001)$ and not significant between lowest and middle tertile $(\mathrm{p}=0.088)$. After adjustment for age, sex, serum albumin, baseline eGFR, blood pressure, and ACEi/ARB treatment, the multivariable adjusted hazard ratio of highest tertile of NAG/C was 18.97 (95\% CI: 1.70, 211.86) compared to lowest tertile (Table S2). The remission rate evaluated in 72 patients with baseline NS was 79\%, 56\% and 29\% in lowest, middle and highest tertile, respectively (Figure S2). The difference was significant between lowest and highest tertile $(\mathrm{p}=0.002)$ and not significant between lowest and middle tertile $(p=0.35)$. Thus the NAG/C highest tertile showed significantly higher progression and lower remission rate compared to lowest tertile.

Functional outcome (progression, remission) according to tertiles of eGFR in all 86 patients. 
The progression rate of lowest (eGFR $<59, \mathrm{n}=28$ ), middle ( $59 \leq$ eGFR $<88, n=29)$, and highest tertile of eGFR (eGFR $\leq 88, n=29)$ were $61 \%, 17 \%$ and $7 \%$, respectively (Figure 4). Compared to highest tertile group, lowest tertile had significantly higher progression rate $(\mathrm{p}<0.001)$ and no significant difference was observed for middle tertile $(\mathrm{p}=0.16)$. After adjustement for age, sex, serum albumin, NAG/C, blood pressure, and ACEi/ARB treatment, the hazard ratio of lowest tertile of eGFR $(<59)$ was 11.58 (95\%CI: 2.02, 66.29) compared to highest tertile (Table S2). The remission rate evaluated in 72 patients with baseline NS was $26 \%$, $61 \%$ and $67 \%$, respectively (Figure S3). The difference was significant between highest and lowest tertile $(\mathrm{p}=0.009)$ and not significant between highest and middle tertile $(p=0.74)$. Thus the lowest eGFR tertile showed higher progression and lower remission rate compared to highest tertile.

\section{Risk stratification}

A risk stratification in two groups was defined by combination of middle and highest tertiles of eGFR ( $\geq 59)$ and lowest and middle tertiles of NAG/C $(<19.2)$ (low risk, $n=43)$ and highest tertile of NAG/C $(\leq 19.2)$ or lowest tertile of eGFR $(<59)$ (high risk, $\mathrm{n}=43$ ). Compared to low risk subjects, high risk subjects had significantly higher progression rate for those with and without NS (Figure S1). For NS subjects, the multivariableadjusted HR of progression for high risk group were 8.62 (95\% CI 1.93, $38.45, \mathrm{p}=0.005)$ compared to low risk group.

Comparison between 30 NS patients IS-untreated (IS-) and 35 NS patients treated with steroids and cyclophosphamide (IS+) according to low and high risk. 
In low risk patients both progression and remission rates were not significantly different between IS- $(n=19)$ and IS+ patients $(n=11)$ : progression: $7 \%$ vs. $18 \%$, respectively $(\mathrm{p}=0.81)$; remission $75 \%$ vs. $73 \%$, respectively $(\mathrm{p}=0.39)$. In high risk patients both progression and remission rates were significantly different between IS- $(n=11)$ vs. IS+ (n $=24)$ : progression $91 \%$ vs. $29 \%(\mathrm{p}=0.003)$; remission $0 \%$ vs. $42 \%$ ( $\mathrm{p}<$ 0.001) (Figure 5, Figure S4). The multivariable-adjusted HR of progression for IS+ was $0.09(95 \%$ CI $0.02,0.45, \mathrm{p}=0.003)$ compared to IS-. Noteworthy were the following observations: in 24 high risk IS+ patients $14(58 \%)$ were unresponsive to treatment: 7 with progression and 7 with persistent nephrotic syndrome with CRF; moreover of 10 high risk patients attaining remission 3 had baseline eGFR <59, while in IS- high risk patients none of them with baseline eGFR $<59$ attained remission.

\section{Change of renal function and proteinuria during follow-up}

period.

In the highest tertile of NAG/C, the median change of eGFR during follow-up period in subjects without IS treatment was -9.1 $\mathrm{ml} / \mathrm{min} / 1.73 \mathrm{~m}^{2} /$ year $\left(25^{\text {th }}, 75^{\text {th }}\right.$ percentile $\left.-13.0,-7.2\right)$ and those with steroids + cyclophosphamide treated was $-0.9 \mathrm{ml} / \mathrm{min} / 1.73 \mathrm{~m}^{2} /$ year $(-2.6$, +1.0 ), which was significantly smaller compared to no-IS subjects ( $\mathrm{p}<$ 0.001) (Table 4). In moderate to lowest tertile of eGFR, the median change of eGFR in no-IS was $-7.8(-10.1,-4.6)$ and $-4.0(-12.8,-0.5)$, and those with steroids + cyclophosphamide was $-1.3(-4.8,+0.2)$ and $0.0(-2.6$, +2.0 ), which was also significantly smaller compared to no-IS subjects ( $\mathrm{p}$ $=0.014$ and 0.038 , respectively). The change of proteinuria during the 
follow-up period in subjects with Steroids + CY were significantly larger compared to those without IS treatments in the moderate eGFR $(59 \leq$ eGFR $<88)$ group.

In the sensitivity analysis with an outcome as incident ESRD, no substantial changes to the result were seen (Data not shown).

\section{Discussion}

In a long follow-up study of 86 IMN subjects, the risk of progression was evaluated for eight baseline urinary markers and renal function. We found that NAG/C and eGFR were independent predictors of progression in both NS and non-NS subjects and allow in combination reliable risk stratification. By risk stratification analysis, high risk NS subjects with IS treatment had significantly lower progression and higher remission rate compared to those without IS treatment. In low risk group there were no significant differences for progression and remission rate between untreated and IS-treated subjects. A recent review of 1.762 IMN patients from 36 randomized controlled trials (20) did not reach definitive conclusions about treatment; combination of steroids and cyclophosphamide appears as the most effective therapy, but is associated with rather high rate of serious adverse events. Recently RTX has been suggested as first line therapy of IMN with NS (13). The superior efficacy of RTX is suggested by its specific pathogenetic mechanism (depletion of $B$ cells responsible of anti-PLA2R antibodies production) and by induction of remission also in patients unresponsive to other immuno-suppressive agents. The high rate of spontaneous remission (about 30-40\%) in IMN patients with NS underlines the importance of availability of baseline risk 
stratification able to identify patient candidates to spontaneous remission and long-lasting NRF for whom supportive therapy is indicated and patients at high risk of progression for whom immuno-suppressive treatment should the first line therapy ideally started at diagnosis and not later in the disease course. A reliable outcome predictor should be based on pathophysiological progression mechanisms and able to predict remission prior to the start of treatment. As well known, proteinuria play a central role in glomerulonephritis $(\mathrm{GN})$ as marker of disease severity, factor responsible of further renal damage at glomerular and tubulointerstitial level (review in 21-24) and outcome predictor; moreover proteinuria reduction by RAS inhibition is associated with lowering of progression to renal failure and improvement of kidney survival (25). It has been demonstrated that tubulo-interstitial damage is also associated with chronic hypoxia (26). Several studies showed that high and low molecular weight (MW) proteins (IgG, IgM, $\alpha 2$-macroglobulin, $\alpha 1$ microglobulin, $\beta 2$-microglobulin) and the lysosomal enzyme NAG have a higher predictive value than albumin/creatinine ratio in various types of primary GN and Diabetic Nephropathy (27-43). Our risk stratification is based on very simple biomarkers such as the tubular damage marker urinary $\mathrm{NAG} / \mathrm{C}$ and eGFR in combination. NAG is a lysosomal enzyme of $130 \mathrm{kDa}$ molecular mass normally excreted in urine in low amounts as a consequence of the normal exocytosis process $(16,43)$; its urinary excretion increases after exposure to several tubulo-toxic substances and in various renal diseases; in glomerular diseases the analysis of NAG isoenzymes showed that the increased urinary excretion is due to an increased release by the renal tubular cells and not to increased filtration 
across the damaged GFB. Thus NAG excretion is universally recognized as a marker of functional and/or structural damage of tubular cells. In a study of 136 patients with IMN, Focal Segmental Glomerulosclerosis (FSGS) and Minimal Change Disease (16) NAG/C showed a significant relationship with 24-hour proteinuria and excretion of $\mathrm{IgG}$ and $\alpha 1 \mathrm{~m}$; in NS patients with NRF NAG/C below or above selected cut-offs significantly predicted remission and progression to ESRD.

The rational for suggestion of immunosuppressive treatment at diagnosis on the basis NAG/C and eGFR risk stratification is based on the observation that high NAG/C or low eGFR NS subjects without immunosuppressive treatment had median renal function decline of -7.8 to $-9.1 \mathrm{ml} / \mathrm{min} / 1.73 \mathrm{~m}^{2} /$ year, which was significantly larger compared to immunosuppressive treated subjects. Thus high risk subjects are suggested for immunosuppressive treatments at diagnosis to preserve renal function, since they are likely to progress during the observational period as recommended in the current guideline (14). In the high risk IS+ patients progression was significantly lower than in IS- patients $(29 \%$ vs. $91 \%, \mathrm{p}=$ 0.003 ) and remission significantly higher (42\% vs. 0\%, p < 0.001$)$, but $58 \%$ of high risk IS+ patients were unresponsive to steroid and cyclophosphamide ( 7 progressors and 7 with persistent nephrotic syndrome after $89 \pm 71$ months from starting therapy); thus an immunosuppressive treatment more effective than steroid and cyclophosphamide is warranted. The low risk IS- patients are characterized by $75 \%$ of remission and $84 \%$ of long-lasting NRF not significantly different from the IS+ patients $(73 \%$ remission and $82 \%$ long-lasting renal function, respectively); thus low risk patients could be considered candidates to 
supportive therapy unless severe NS is present at risk of complications. On the basis of our data that reliably identify low and high risk patients may be rational the proposal of supportive therapy for low risk subjects and Rituximab as first-line therapy at diagnosis and not later after unresponsiveness to other treatments for high risk patients as more specific, safer and effective agent also in patients unresponsive to other non-specific treatments often associated with serious adverse events.

\section{Conclusions}

The risk stratification based on urinary NAG/C and eGFR, markers of pathophysiological progression mechanisms, is very simple, low costly and readily available in clinical practice and may be a reliable criterion to identify at diagnosis the IMN patients with high probability of remission and long-lasting NRF or at risk of progression and thus may be a useful tool for early therapeutic decisions and selection of patients which should be treated with supportive therapy or with RTX as first-line therapy at diagnosis and not later after unresponsiveness to other treatments. 
Conflict of interest: The authors declare that they have not conflict of interest. 


\section{References}

1) Ronco P, Debiec H: Pathophysiological advances in membranous nephropathy: time for a shift in patient's care. Lancet 2015

2) $\mathrm{Du} \mathrm{Y}, \mathrm{Li}$ J, He F et al: The diagnosis accuracy of PLA2R-AB in the Diagnosis of Idiopathic Membranous Nephropaty: A Meta-Analysis. PloS One 2014; 9: 104936:

3) Oh YJ, Yang SH, Kim DH, Kang SW, Kim IS: Autoantibodies agaist phospholipase A2 receptor in Korean patients with membranous nephropathy. PLoS One 2013; 8: e62151.

4) Lv J, Hou W, Zhou X, et al: Interaction between PLA2R1 and HLA-DQA1 variants associates with anti-PLA2R antibodies and membranous nephropathy. $J$ Am Soc Nephrol 2013; 24: 1323-29

5) Bech AP, Hofstra JM, Brenchley PE, Wetzels JF: Association of anti-PLA2R antibodies with outcomes after immunosuppressive therapy in idiopathic membranous nephropathy. Clin J Am Soc Nephrol 2014; 9:1386-92.

6) Hoxha E, Thiele I, Zahner G, Panzer U, Harendza S, Stahl RA: Phospholipase A2 receptor autoantibodies and clinical outcome in patients with primary membranous nephropathy. J Am Soc Nephrol 2014; 25: 1357-66.

7) Hoxha E, Harendza S, Pinnschmidt H, Panzer U, Stahl RA: PLA2R Antibody Levels and Clinical Outcome in Patients with Membranous Nephropathy and Non-Nephrotic Range Proteinuria under Treatment with Inhibitors of the Renin-aangiotensin System. PLoS One 2014; 9: e110681.

8) Hoxha E, Harendza S, Pinnschmidt H, Panzer U, Stahl RA: M-type phospholipase A2 receptor autoantibodies and renal function in patients with primary membranous nephropathy. Clin J Am Soc Nephrol 2014; 9: 1883-1890.

9) Beck LH jr, Fervenza FC, Beck DM, et al: Rituximab induced depletion of anti PLA2R autoantibodies predict response in membranous nephropathy. J Am SocNephrol 2011; 22:1543-50.

10) Ruggenenti P, Cravedi $P$, Chianca $A$ et al: Rituximab in Idiopathic Membranous Nephropathy. J Am Soc Nephrol 2012; 23: 1416-1425.

11) Fervenza FC, Canetta PA, Barbour SJ et al: A Multicenter Randomized Controlled Trial of Rituximab versus Cyclosporine in the Treatment of Idiopathic Membranous Nephropathy (MENTOR). Nephron 2015; 130: 158-168 
12) Ruggenenti $P$, Debiec $H$, Ruggiero $B$, et al: Anti-phospholipase A2 receptor antibody titer predicts post-rituximab outcome of membranous nephropathy. J Am Soc Nephrol 2015; 26: 2545-2558

13) Cravedi P, Remuzzi G, Ruggenenti P: Rituximab in Primary Membranous Nephropathy: First-Line Therapy, Why Not ? Nephron Clin Pract 2014; 128: 261-269

14) KIDNEY DISEASE IMPROVING GLOBAL OUTCOMES. KDIGO Clinical Practice Guideline for Glomerulonephritis. Chapter 7: Idiopathic membranous nephropathy. Kidney Int Suppl. $2012 ; 2: 186-197$

15) Bazzi C, Petrini C, Rizza V, Napodano P, Paparella M, Arrigo G, Pisano L, D’Amico G: Fractional excretion of IgG predicts renal outcome and response to therapy in primary focal segmental glomerulosclerosis: a pilot study. Am J Kidney Dis 2003, 41(2):328-335

16) Bazzi C, Petrini C, Rizza V, Arrigo G, Napodano P, Paparella M, D'Amico G : Urinary $\mathrm{N}$-acetyl- $\beta$-glucosaminidase excretion is a marker of tubular cell dysfunction and predictor of outcome in primary glomerulonephritis. Nephrol Dial Transplant 2002; $17: 1890-1896$

17) Levey AS, Stevens LA Schmid CH, Zhang YL, Castro AF 3rd, Feldman, HI, Kusek JW, Eggers P, Van Lente F, Greene T, Coresh J; CKD-EPI (Chronic Kidney Disease Epidemiology Collaboration). A new equation to estimate glomerular filtration rate. Ann Intern Med. 2009; 150: 604-612

18) D’Amico G, Ferrario F, Colasanti G, Ragni A, Bestetti Bosisio M: IgA-mesangial nephropathy (Berger's disease) with rapid decline in renal function. Clin Nephrol. 1981; 16: 251-257

19) Ponticelli C, Altieri P, Scolari F et al: A randomized study comparing methylprednisolone plus clorambucil versus methylprednisolone plus cyclophosphamide in idiopathic membranous nephropathy. J Am Soc Nephrol.1998; 9: 444-450.

20) Chen Y, Schieppati A, Cai G et al: Immunosuppression for membranous nephropathy: a systematic review and meta-analysis of $\mathbf{3 6}$ clinical trials. Clin $\mathrm{J}$ Am Soc Nephrol 2013; 8: 787-796

21) Remuzzi G, Benigni A, Remuzzi A: Mechanisms of progression and regression of renal lesions of chronic nephropathies and diabetes. J Clin Invest 2005; 116: 288-296 
22) Ruggenenti P, Cravedi P, Remuzzi G: Mechanism and Treatment of CKD. J Am Soc Nephrol 2012; 23: 1917-1928.

23) Cravedi P, Remuzzi G: Pathophysiology of proteinuria and its value as outcome measure in CKD. Br J Clin Pharmacol 2013; 76: 516-523

24) Zoja C, Abbate M, Remuzzi G. Progression of renal injury toward interstitial inflammation and glomerular sclerosis is dependent on abnormal protein filtration. Nephrol Dial Transplant 2015; 30:706-712

25) van der Meer I, Cravedi P, Remuzzi G. The role of renin angiotensin system inhibition in kidney repair. Fibrogenesis Tissue Repair 2010; 3:7

26) Nangaku M: Chronic Hypoxia and Tubulointerstitial Injury: A Final Common Pathway to End-Stage Renal Failure. J Am Soc Nephrol 2006; 17: 17-25

27) Irazabal MW, Eirin A, Lieske J et al: Low- and high-molecular weight proteins as predictors of response to rituximab in patients with membranous nephropathy: a prospective study. Nephrol Dial Transplant 2013; 28: 137-146.

28) Bakoush O, Torffvit O, Rippe B, Tencer J: High proteinuria selectivity index based upon IgM is a strong predictor of poor renal survival in glomerular diseases. Nephrol Dial, Transplant 2001;16: 1357-1363.

29) Bakoush O, Segelmark M, Torffvit O, Ohlsson S, Tencer J: Urine IgM excretion predicts outcome in ANCA-associated renal vasculitis. Nephrol Dial Transplant 2006; 21:1263-1269.

30) Tofik R, Torffvit O, Tippe B, Bakoush O: Increased urine IgM excretion predicts cardiovascular events in patients with type 1 diabetic nephropathy. BMC Med 2009. doi. 10. 1186/1741-7015-7-39

31) Tofik R, Aziz R, Reda A wt al: The value of IgG-uria in predicting renal failure in idiopathic glomerular diseases. A long-term follow up study. Scand J Clin Lab Invest 2011; 71: 123-128 
32) Bakoush O, Torffvit O, Rippe B, Tencer J: Renal function in proteinuric glomerular diseases correlates to the changes in urine $\operatorname{IgM}$ excretion but not to the changes in the degree of albuminuria. Clin Nephrol 2003; 59: 345-352.

33) Bakoush O, Tencer J, Tapia J, Rippe B, Torffvit O: Higher urinary IgM excretion in type 2 diabetic nephropathy compared to type 1 diabetic nephropathy. Kidney Int 2002; 61: 203-208.

34) Bazzi C, Petrini C, Rizza V et al: A modern approach to selectivity of proteinuria and tubulointerstitial damage in nephrotic syndrome. Kidney Int 2000; 58: 1732-1741

35) Bazzi C, Rizza V, Raimondi S et al: In Crescentic IgA Nephropathy Fractional Excretion of IgG in combination with Nephron Loss is the Best Predictor of Progression and Responsiveness to Immunosuppression. Clin J Am Soc Nephrol 2009; 4: $929-935$

36) Bazzi C, Rizza V, Casellato D et al: Validation of some pathophysiological mechanisms of the CKD progression theory and outcome prediction in IgA nephropathy. J Nephrol 2012; 25:810-818

37) Bazzi C, Rizza V, Casellato D et al: Urinary IgG and $\boldsymbol{\alpha 2}$-macroglobulin are powerful predictors of outcome and responsiveness to steroids and cyclophosphamide in idiopathic focal segmental glomerulosclerosis with nephrotic syndrome. Biomed Res Int 2013: doi:10.1155/2013/941831

38) Mc Quarrie EP, Shakerdi L, Jardine AG et al: Fractional excretions of albumin and IgG are the best predictors of progression in primary glomerulonephritis. Nephrol Dial Transplant 2011; 26: 1563-1569.

39) Bazzi C, Rizza V, Casellato D, Tofik R, Berg AL, Gallieni M, D'Amico G, Bakoush O: Fractional excretion of IgG in idiopathic membranous nephropathy with nephrotic syndrome: a predictive marker of risk and drug responsiveness. BMC Nephrol 2014; doi: 10. 1186/1471-2369-15-74

40) Branten AJ, du BUF-Vereijken, Klasen IS, Bosch FH, Feith GW, Hollander DA, 
Wetzels JF: Urinary excretion of beta2-microglobulin and IgG predict prognosis in idiopathic membranous nephropathy: a validation study. J Am Soc Nephrol 2005; $16: 169-174$

41) Jan AJG, van den Brand JAJG, Hofstra JM, Wetzels JFM: Low-Molecular-Weight Proteins as Prognostic Markers in Idiopathic Membranous Nephropathy. Clin J Am Soc Nephrol 2011; 6: 2846-2853

42) Jan AJG, van den Brand JA, Hofstra JM, Wetzels JFM: Prognostic Value of Risk Score and Urinary Markers in Idiopathic Membranous Nephropathy. Clin J Am Soc Nephrol 2012; 7: 1242-1248

43) D’Amico G, Bazzi C: Urinary protein and enzyme excretion as markers of tubular damage. Curr Opin Nephrol Hypertens 2003; 38 (Suppl): 14-19 



\section{LEGENDS FOR TABLES AND FIGURES}

Table 1. Baseline characteristics of study subjects $(n=86)$

Age, serum albumin, and baseline eGFR are mean (SD). Male, BP, and ACEi treatments are subject numbers (percentage). Urinary markers are geometric means (95\% confidence interval). Follow-up are median (range).

Abbreviations: BP, blood pressure; eGFR, estimated glomerular filtration rate; $\mathrm{UP} / \mathrm{C}$, total urinary proteins/ $1 \mathrm{~g}$ urinary creatinine; $\alpha 1 \mathrm{~m} / \mathrm{C}$, urinary $\alpha 1$-microglobulin/ $1 \mathrm{~g}$ urinary creatinine; NAG/C, urinary $\mathrm{N}$-acetyl- $\beta$-glucosaminidase $/ 1 \mathrm{~g}$ urinary creatinine; $\alpha 2 \mathrm{~m} / \mathrm{C}$, urinary $\alpha 2$-macroglobulin $/ 1 \mathrm{~g}$ urinary creatinine; Tf/C, serum Transferrin/1 g urinary creatinine; $\mathrm{IgG} / \mathrm{C}$, urinary $\mathrm{IgG} / 1 \mathrm{~g}$ urinary creatinine; $\mathrm{ACEi}$, angiotensin converting enzyme inhibitor; $\mathrm{ARB}$, angiotensin II receptor; SD, standard deviation.

Table 2. Area under the receiver operating characteristic curves for prediction of progression by urinary markers

(A) Proteinuria, UP/C, $\alpha 1 \mathrm{~m} / \mathrm{C}, \mathrm{NAG} / \mathrm{C}, \mathrm{Tf} / \mathrm{C}$, and $\mathrm{IgG} / \mathrm{C}(\mathrm{n}=86)$. (B) Proteinuria and $\alpha 2 \mathrm{~m} / \mathrm{C}(\mathrm{n}=$ 45).

Abbreviations: UP/C, total urinary proteins/g urinary creatinine; $\alpha 1 \mathrm{~m} / \mathrm{C}$, urinary $\alpha 1$-microglobulin $/ \mathrm{g}$ urinary creatinine; NAG/C, urinary $\mathrm{N}$-acetyl- $\beta$-glucosaminidase/g urinary creatinine; $\mathrm{Tf} / \mathrm{C}$, serum Transferrin/g urinary creatinine; IgG/C, urinary IgG/g urinary creatinine; $\alpha 2 \mathrm{~m} / \mathrm{C}$, urinary $\alpha 2$ macroglobulin/g urinary creatinine.

Table 3. Unadjusted and multivariable-adjusted hazard ratio for progression $(n=86)$

*Adjusted for age, sex, serum albumin, baseline eGFR, blood pressure, and ACE inhibitor treatment. Abbreviations: BP, blood pressure; eGFR, estimated glomerular filtration rate; UP/C, total urinary proteins/g urinary creatinine; $\alpha 1 \mathrm{~m} / \mathrm{C}$, urinary $\alpha 1$-microglobulin/g urinary creatinine; NAG/C, urinary $\mathrm{N}$-acetyl- $\beta$-glucosaminidase/g urinary creatinine; $\alpha 2 \mathrm{~m} / \mathrm{C}$, urinary $\alpha 2$-macroglobulin/g urinary creatinine; Tf/C, serum Transferrin/g urinary creatinine; IgG/C, urinary IgG/g urinary creatinine; ACEi, angiotensin converting enzyme inhibitor; ARB, angiotensin II receptor blocker.

Table 4. Median (25th, 75 th percentile) of eGFR changes $\left(\mathrm{ml} / \mathrm{min} / 1.73 \mathrm{~m}^{2} /\right.$ year$)$ by nephrotic syndrome and treatment according to tertiles of NAG/C or eGFR $(n=86)$

eGFR change $=[$ eGFR(last $)-$ eGFR $(1$ st $)] /$ Follow-up $\times 12$

Abbreviations: NAG/C, urinary N-acetyl- $\beta$-glucosaminidase/1 g urinary creatinine; eGFR, estimated glomerular filtration rate. 
Figure 1. Flow charts of study subjects for the analysis $(n=86)$

Figure 2. Receiver Operating Characteristic curves for prediction of progression by urinary markers

(A) Proteinuria, UP/C, $\alpha 1 \mathrm{~m} / \mathrm{C}, \mathrm{NAG} / \mathrm{C}, \mathrm{Tf} / \mathrm{C}, \mathrm{IgG} / \mathrm{C}(\mathrm{n}=86)$. (B) Proteinuria, $\alpha 2 \mathrm{~m} / \mathrm{C}(\mathrm{n}=45)$.

Abbreviations: $\mathrm{UP} / \mathrm{C}$, total urinary proteins $/ \mathrm{g}$ urinary creatinine; $\alpha 1 \mathrm{~m} / \mathrm{C}$, urinary $\alpha 1$-microglobulin $/ \mathrm{g}$ urinary creatinine; NAG/C, urinary $\mathrm{N}$-acetyl- $\beta$-glucosaminidase/g urinary creatinine; $\mathrm{Tf} / \mathrm{C}$, serum Transferrin/g urinary creatinine; IgG/C, urinary IgG/g urinary creatinine; $\alpha 2 \mathrm{~m} / \mathrm{C}$, urinary $\alpha 2$ macroglobulin/g urinary creatinine.

Figure 3. Kaplan-Meier curves for the percentages of subjects with progression tertiles of $\mathrm{NAG} / \mathrm{C}(\mathbf{n}=\mathbf{8 6})$

Abbreviation: NAG/C, urinary N-acetyl- $\beta$-glucosaminidase/g urinary creatinine.

Figure 4. Kaplan-Meier curves for the percentages of subjects with progression by tertiles of eGFR $(n=86)$

Abbreviation: eGFR, estimated glomerular filtration rate.

Figure 5. Kaplan-Meier curves for the percentages of subjects with progression with or without immunosuppressive treatment in low and high risk nephrotic syndrome subjects $(\mathrm{n}=$ 65)

(A) Low risk (NAG/C $<19.2$ and 59 $\leq$ eGFR) nephrotic syndrome subjects $(\mathrm{n}=30)$. (B) High risk $(19.2 \leq \mathrm{NAG} / \mathrm{C}$ or eGFR $<59)$ nephrotic syndrome subjects $(\mathrm{n}=35)$.

Abbreviations: NAG/C, urinary N-acetyl- $\beta$-glucosaminidase/g urinary creatinine; eGFR, estimated glomerular filtration rate; IS, immunosuppressive treatment; CY, Cyclophosphamide. 


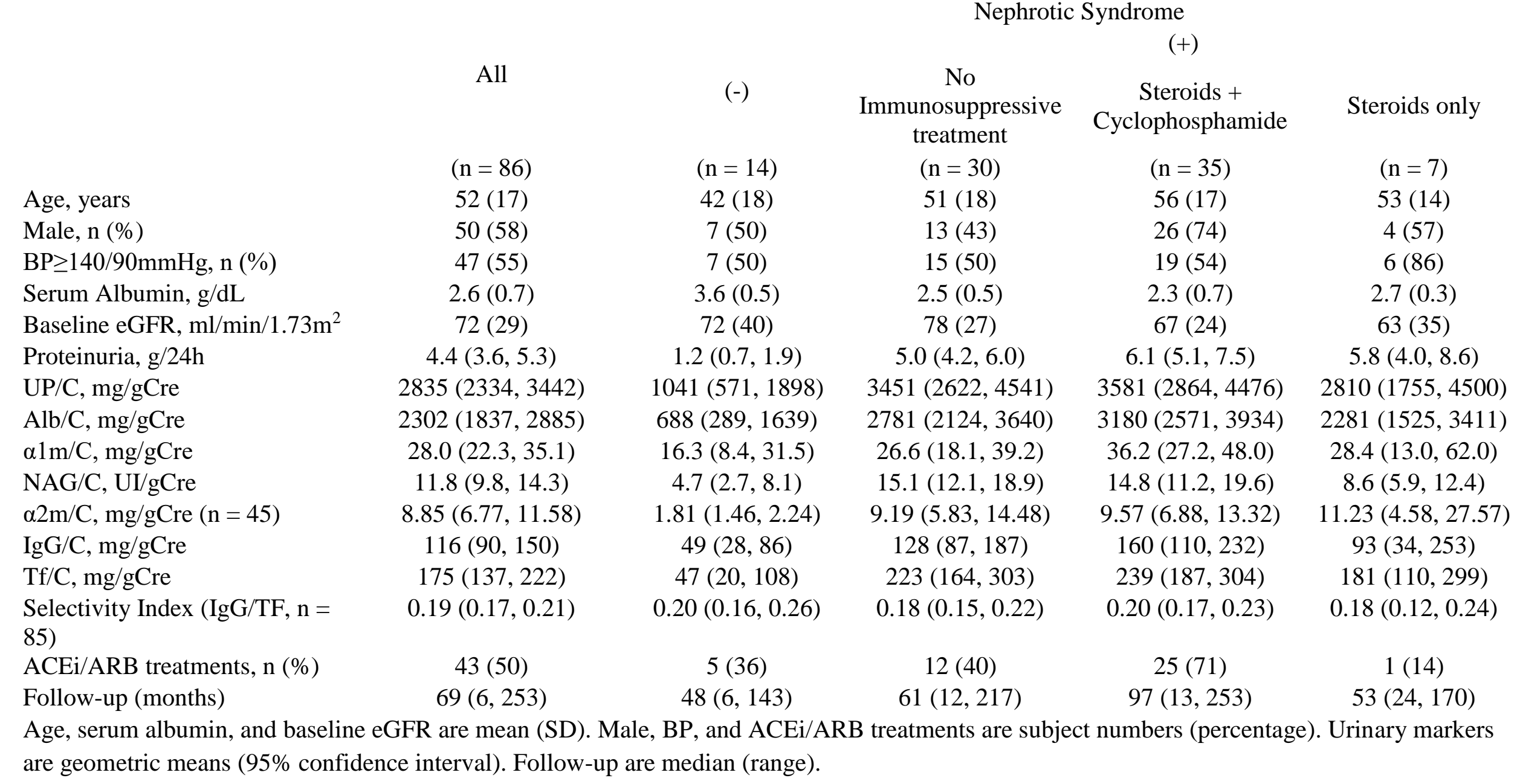


Table 2. Area under the receiver operating characteristic curves for prediction of progression by urinary markers

A. $(\mathbf{n}=86)$

\begin{tabular}{lccc}
\hline & Area Under Curve & $95 \%$ CI & P \\
\hline Proteinuria & 0.637 & $(0.509,0.765)$ & - \\
$\mathrm{UP} / \mathrm{C}$ & 0.748 & $(0.626,0.870)$ & 0.012 \\
$\mathrm{Alb} / \mathrm{C}$ & 0.733 & $(0.605,0.862)$ & 0.034 \\
$\alpha 1 \mathrm{~m} / \mathrm{C}$ & 0.785 & $(0.685,0.886)$ & 0.021 \\
$\mathrm{NAG} / \mathrm{C}$ & 0.770 & $(0.668,0.860)$ & 0.018 \\
$\mathrm{IgG} / \mathrm{C}$ & 0.754 & $(0.649,0.860)$ & 0.051 \\
$\mathrm{Tf} / \mathrm{C}$ & 0.702 & $(0.570,0.835)$ & 0.20 \\
\hline
\end{tabular}

B. $(\mathbf{n}=\mathbf{4 5})$

\begin{tabular}{lccc}
\hline & Area Under Curve & $95 \%$ CI & $\mathrm{P}$ \\
\hline Proteinuria & 0.600 & $(0.425,0.775)$ & - \\
$\alpha 2 \mathrm{~m} / \mathrm{C}$ & 0.526 & $(0.337,0.715)$ & 0.37 \\
\hline
\end{tabular}


Table 3. Unadjusted and multivariable-adjusted hazard ratio for progression $(n=86)$

\begin{tabular}{|c|c|c|c|c|c|c|}
\hline & \multicolumn{6}{|c|}{ Hazard Ratio } \\
\hline & Unadjusted & $(95 \% \mathrm{CI})$ & $\mathrm{p}$ & Multivariable-adjusted* & $(95 \% \mathrm{CI})$ & $\mathrm{p}$ \\
\hline Age, years & 1.03 & $(1.00,1.05)$ & 0.023 & 0.98 & $(0.94,1.01)$ & 0.10 \\
\hline Male vs. Female & 1.03 & $(0.46,2.34)$ & 0.94 & 0.64 & $(0.27,1.56)$ & 0.33 \\
\hline Serum Albumin, g/dL & 0.77 & $(0.43,1.37)$ & 0.37 & 0.49 & $(0.25,0.97)$ & 0.039 \\
\hline Baseline eGFR, $\mathrm{ml} / \mathrm{min} / 1.73 \mathrm{~m}^{2}$ & 0.97 & $(0.95,0.98)$ & $<0.001$ & 0.96 & $(0.94,0.98)$ & $<0.001$ \\
\hline $\mathrm{BP} \geq 140 / 90 \mathrm{mmHg}$ & 3.88 & $(1.52,9.86)$ & 0.004 & 2.54 & $(0.62,10.36)$ & 0.19 \\
\hline ACEi/ARB treatments & 0.30 & $(0.13,0.72)$ & 0.007 & 0.23 & $(0.09,0.57)$ & 0.002 \\
\hline Proteinuria $\times 10$ & 2.28 & $(0.53,9.85)$ & 0.27 & 1.08 & $(0.15,7.86)$ & 0.11 \\
\hline $\mathrm{UP} / \mathrm{C} \times 10$ & 14.65 & $(2.36,91.02)$ & 0.004 & 4.71 & $(0.79,28.12)$ & 0.089 \\
\hline $\mathrm{Alb} / \mathrm{C} \times 10$ & 11.36 & $(1.73,74.59)$ & 0.011 & 3.95 & $(0.75,20.90)$ & 0.11 \\
\hline$\alpha 1 \mathrm{~m} / \mathrm{C} \times 10$ & 10.41 & $(3.36,32.28)$ & $<0.001$ & 2.29 & $(0.45,11.70)$ & 0.32 \\
\hline $\mathrm{NAG} / \mathrm{C} \times 10$ & 16.98 & $(3.21,89.77)$ & 0.001 & 20.98 & $(2.70,162.69)$ & 0.004 \\
\hline $\mathrm{IgG} / \mathrm{C} \times 10$ & 5.85 & $(2.17,15.77)$ & $<0.001$ & 1.86 & $(0.50,6.97)$ & 0.36 \\
\hline $\mathrm{Tf} / \mathrm{C} \times 10$ & 6.17 & $(1.32,28.93)$ & 0.021 & 2.80 & $(0.70,11.23)$ & 0.15 \\
\hline$\alpha 2 \mathrm{~m} / \mathrm{C} \times 10$ & 1.48 & $(0.37,5.84)$ & 0.57 & 0.14 & $(0.02,1.13)$ & 0.065 \\
\hline
\end{tabular}

* Adjusted for age, sex, serum albumin, baseline eGFR, blood pressure, and ACEi/ARB treatment. 
Table 4. Median (25th, 75th percentile) of eGFR changes ( $\mathrm{ml} / \mathrm{min} / 1.73 \mathrm{~m}^{2} /$ year) by nephrotic syndrome and treatment according to tertiles of NAG/C or eGFR $(n=86)$

Nephrotic Syndrome

$(-)$

$\begin{array}{lcc} & & \begin{array}{c}\text { No Immunosuppressive } \\ \text { treatment } \\ (\mathrm{n}=30)\end{array} \\ \mathrm{NAG} / \mathrm{C}<9.4 & (\mathrm{n}=14) & -3.0(-3.7,-1.2) \\ 9.4 \leq \mathrm{NAG} / \mathrm{C}<19.2 & -6.4(-2.0,+3.6) & -1.7(-3.6,0.0) \\ 19.2 \leq \mathrm{NAG} / \mathrm{C} & -2.0 & -9.1(-13.0,-7.2) \\ \mathrm{eGFR}<59 & -2.0(-11.1,-1.8) & -7.8(-10.1,-4.6) \\ 59 \leq \mathrm{eGFR}<88 & +16.0 & -4.0(-12.8,-0.5) \\ 88 \leq \mathrm{eGFR} & -1.0(-2.0,+1.6) & -1.9(-3.3,-1.2) \\ \text { eGFR change }=[\text { eGFR }(\text { last })-\text { eGFR }(1 \mathrm{st})] / \text { Follow-up } \times 12\end{array}$

eGFR change $=[$ eGFR(last $)-$ eGFR $(1 \mathrm{st})] /$ Follow-up $\times 12$
$(+)$

No Immunosuppressive treatment vs. Steroids + Cyclophosphamide

Steroids + Cyclophosphamide $(\mathrm{n}=35)$ $-0.1(-3.7,+0.4)$ $-0.7(-4.0,+0.4)$ $-0.9(-2.6,+1.0)$ $-1.3(-4.8,+0.2)$ $0.0(-2.6,+2.0)$ $-0.9(-4.0,+0.2)$ $\mathrm{p}$

Steroids only

$\begin{array}{cc}(\mathrm{n}=7) & \\ -0.7(-4.2,+1.9) & 0.48 \\ +0.7(-0.01,+1.5) & 0.66 \\ +0.8 & <0.001 \\ +0.8(-0.1,+1.5) & 0.014 \\ -1.6(-6.8,+3.5) & 0.038 \\ -0.7(-1.6,+0.2) & 0.69\end{array}$




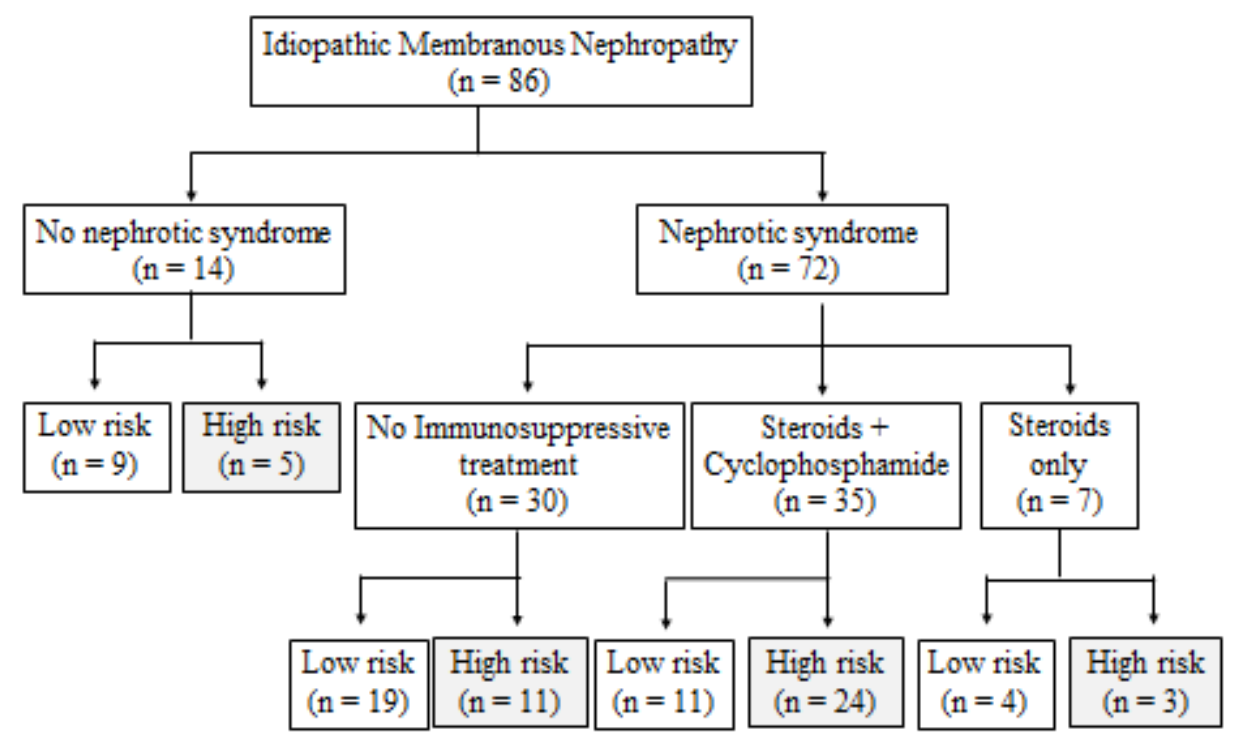

Figure 1. Flow charts of study subjects for the analysis $(n=86)$

(A)

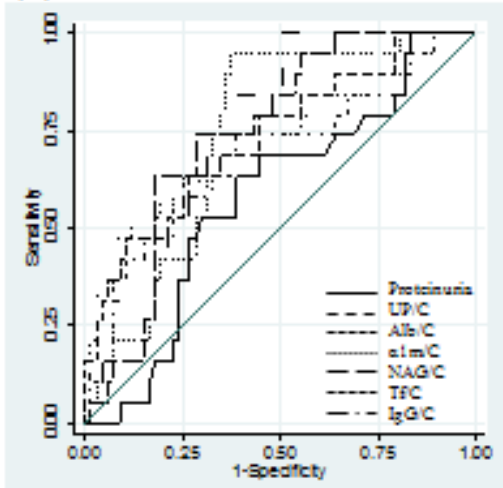

(B)

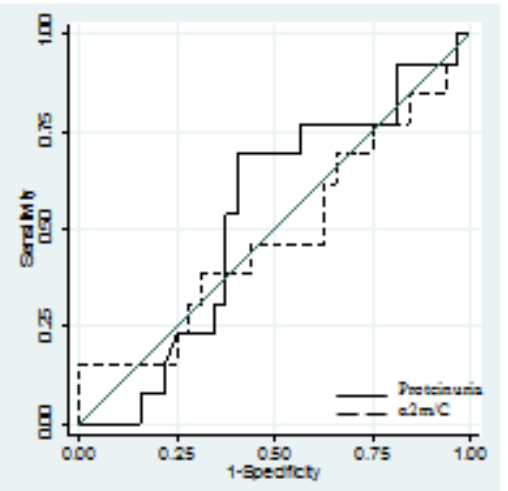

Figure 2. Receiver Operating Characteristic curves for prediction of progression by urinary markers

(A) Proteinuria, UP/C, $\alpha 1 \mathrm{~m} / \mathrm{C}, \mathrm{NAG} / \mathrm{C}, \mathrm{Tf} / \mathrm{C}$, and $\mathrm{IgG} / \mathrm{C}(\mathrm{n}=86)$

(B) Proteinuria and $\alpha 2 \mathrm{~m} / \mathrm{C}(\mathrm{n}=45)$ 


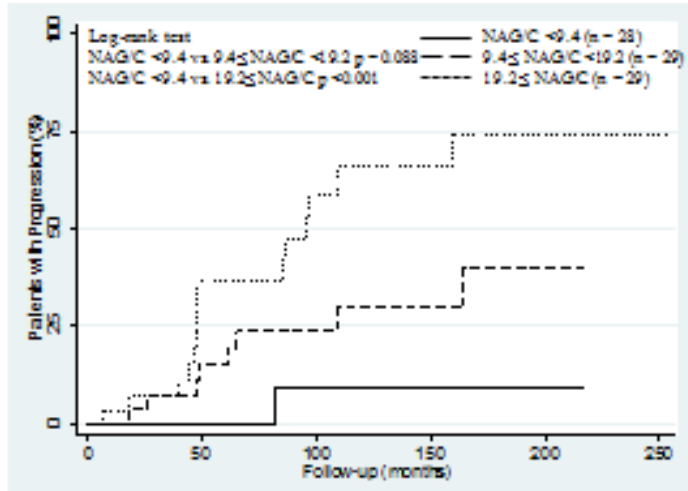

Figure 3. Kaplan-Meier curves for the percentages of subjects with progression tertiles of $\mathrm{NAG} / \mathrm{C}(\mathrm{n}=86)$

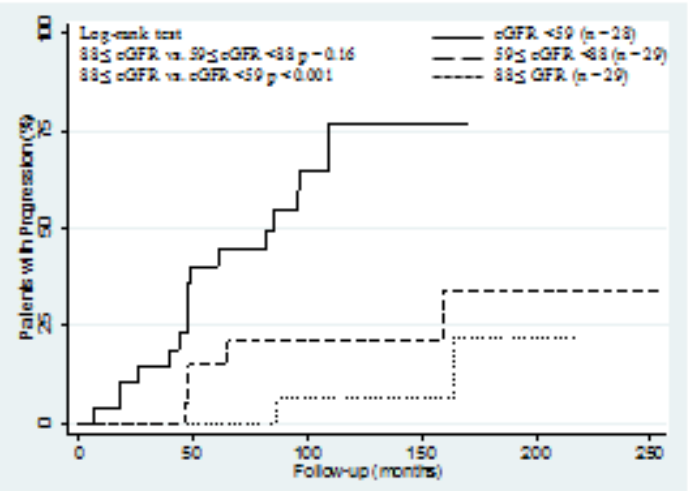

Figure 4. Kaplan-Meier curves for the percentages of subjects with progression by tertiles of eGFR $(n=86)$ 
(A)

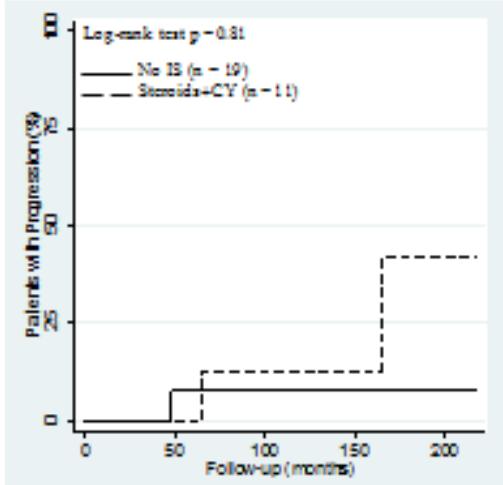

(B)

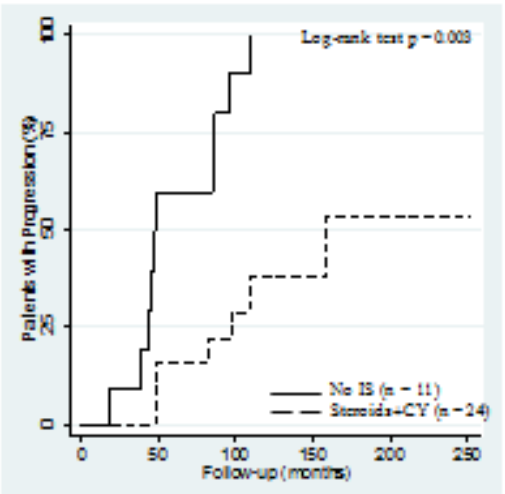

Figure 5. Kaplan-Meier curves for the percentages of subjects with progression with or without immunosuppressive treatment in low and high risk nephrotic syndrome subjects $(n=65)$

(A) Low risk (NAG/C $<19.2$ and $59 \leq$ eGFR) nephrotic syndrome subjects ( $n=30$ )

(B) High risk $(19.2 \leq \mathrm{NAG} / \mathrm{C}$ or eGFR $<59)$ nephrotic syndrome subjects ( $\mathrm{n}=35$ ) 


\section{LEGENDS FOR SUPPLEMENTARY TABLES AND FIGURES}

Table S1. Number of subjects by renal function and clinical remission status at last observation $(n=86)$

Progression: ESRD or eGFR reduction $\geq 50 \%$ of baseline value.

Abbreviations: eGFR, estimated glomerular filtration rate; ESRD, end-stage renal disease.

Table S2. Multivariable-adjusted hazard ratio for progression by tertiles of NAG/C or eGFR $(\mathbf{n}=86)$

a) Adjusted for age, sex, serum albumin, baseline eGFR, blood pressure, and ACEi/ARB treatment.

b) Adjusted for age, sex, serum albumin, NAG/C, blood pressure, and ACEi/ARB treatment.

Abbreviations: $\mathrm{BP}$, blood pressure; eGFR, estimated glomerular filtration rate; UP/C, total urinary proteins $/ 1 \mathrm{~g}$ urinary creatinine; $\alpha 1 \mathrm{~m} / \mathrm{C}$, urinary $\alpha 1$-microglobulin/ $1 \mathrm{~g}$ urinary creatinine; NAG/C, urinary $\mathrm{N}$-acetyl- $\beta$-glucosaminidase $/ 1 \mathrm{~g}$ urinary creatinine; $\alpha 2 \mathrm{~m} / \mathrm{C}$, urinary $\alpha 2$-macroglobulin/ $1 \mathrm{~g}$ urinary creatinine; $\mathrm{Tf} / \mathrm{C}$, serum Transferrin/1 $\mathrm{g}$ urinary creatinine; $\mathrm{IgG} / \mathrm{C}$, urinary $\mathrm{IgG} / 1 \mathrm{~g}$ urinary creatinine; ACEi, angiotensin converting enzyme inhibitor; ARB, angiotensin II receptor blocker.

Table S3. Median (25th, 75th percentile) of proteinuria changes (g/24h) of subjects with nephrotic syndrome by treatments according to tertiles of NAG/C or eGFR $(n=72)$

Proteinuria change $=$ Proteinuria $($ last $)-$ Proteinuria $(1 \mathrm{st})$

Abbreviations: NAG/C, urinary N-acetyl- $\beta$-glucosaminidase/1 g urinary creatinine; eGFR, estimated glomerular filtration rate.

Figure S1. Kaplan-Meier curves for the percentages of subjects with progression with and without nephrotic syndrome $(\mathbf{n}=86)$

(A) Subjects without nephrotic syndrome (n=14). (B) Subjects with nephrotic syndrome $(n=72)$.

Figure S2. Kaplan-Meier curves for the percentages of nephrotic syndrome subjects with clinical remission by tertiles of $\mathrm{NAG} / \mathrm{C}(\mathrm{n}=72)$

Abbreviation: NAG/C, urinary $\mathrm{N}$-acetyl- $\beta$-glucosaminidase/1 g urinary creatinine.

Figure S3. Kaplan-Meier curves for the percentages of nephrotic syndrome subjects with clinical remission by tertiles of eGFR $(n=72)$ 
Abbreviation: eGFR, estimated glomerular filtration rate.

Figure S4. Kaplan-Meier curves for the percentages of subjects with clinical remission with or without immunosuppressive treatment in low and high risk nephrotic syndrome subjects (n = 65)

(A) Low risk (NAG/C $<19.2$ and 59 $\leq$ GGF) nephrotic syndrome subjects $(\mathrm{n}=30)$. (B) High risk $(19.2 \leq \mathrm{NAG} / \mathrm{C}$ or eGFR $<59)$ nephrotic syndrome subjects $(\mathrm{n}=35)$

Abbreviations: NAG/C, urinary N-acetyl- $\beta$-glucosaminidase/1 g urinary creatinine; eGFR, estimated glomerular filtration rate; IS, immunosuppressive treatment; CY, Cyclophosphamide. 
Table S1. Number of subjects by renal function and clinical remission status at last observation $(\mathrm{n}=86)$

Nephrotic Syndrome at baseline

$(+)$

$(-)$

$$
(\mathrm{n}=14)
$$

Last observation

$9(64)$

ormal renal function (eGFR $\geq 60$ )

Clinical remission (+)

Clinical remission (-)

Chronic renal failure $(15<\mathrm{eGFR}<60)$

Clinical remission $(+)$

Clinical remission (-)

End-stage renal disease $(\mathrm{eGFR} \leq 15)$

eGFR reduction $\geq 50 \%$ of baseline

Progression

Progression: ESRD or eGFR reduction $\geq 50 \%$ of baseline value.

\section{No} Immunosuppressive treatment

$(\mathrm{n}=30)$

Steroids + Cyclophosphamide

$(\mathrm{n}=35)$

$14(40)$

5 (14)

$4(11)$

5 (14)

7 (20)

9 (26)

9 (26) n. of subjects $(\%)$
Steroids only

$$
(\mathrm{n}=7)
$$

1 (14)

$0(0)$

$0(0)$

$0(0)$ 
Table S2. Multivariable-adjusted Hazard Ratio for progression by tertiles of NAG/C or eGFR $(n=86)$

\begin{tabular}{lccc}
\hline & Hazard Ratio & $(95 \% \mathrm{CI})$ & $\mathrm{p}$ \\
\hline $\mathrm{NAG} / \mathrm{C}<9.4$ & 1.00 (reference) & - & - \\
$9.4 \leq \mathrm{NAG} / \mathrm{C}<19.2^{\mathrm{a})}$ & 9.81 & $(0.97,99.60)$ & 0.053 \\
$19.2 \leq \mathrm{NAG} / \mathrm{C}^{\mathrm{a})}$ & 18.97 & $(1.70,211.86)$ & 0.017 \\
$\mathrm{eGFR}<59^{\mathrm{b})}$ & 11.58 & $(2.02,66.29)$ & 0.006 \\
$59 \leq \mathrm{eGFR}<88^{\mathrm{b})}$ & 2.77 & $(0.39,19.41)$ & 0.31 \\
$88 \leq \mathrm{eGFR}$ & 1.00 (reference) & - & - \\
\hline
\end{tabular}

a) Adjusted for age, sex, serum albumin, baseline eGFR, blood pressure, and ACEi/ARB treatment.

b) Adjusted for age, sex, serum albumin, NAG/C, blood pressure, and ACEi/ARB treatment. 
Table S3. Median (25th, 75th percentile) of proteinuria changes ( $\mathrm{g} / 24 \mathrm{hr}$ ) of subjects with nephrotic syndrome by treatment according to tertiles of NAG/C or eGFR $(n=72)$

\begin{tabular}{|c|c|c|c|c|}
\hline & \multicolumn{3}{|c|}{ Nephrotic Syndrome } & \multirow{2}{*}{$\begin{array}{c}\text { No Immunosuppressive } \\
\text { treatment vs. Steroids }+ \\
\text { Cyclophosphamide } \\
\text { p }\end{array}$} \\
\hline & $\begin{array}{c}\text { No Immunosuppressive } \\
\text { treatment } \\
(\mathrm{n}=30)\end{array}$ & $\begin{array}{c}\text { Steroids }+ \\
\text { Cyclophosphamide } \\
(\mathrm{n}=34)\end{array}$ & $\begin{array}{l}\text { Steroids only } \\
\qquad(\mathrm{n}=7)\end{array}$ & \\
\hline $\mathrm{NAG} / \mathrm{C}<9.4$ & $-2.2(-3.8,-1.0)$ & $-3.3(-3.9,-3.1)$ & $-1.9(-4.3,+1.9)$ & 0.29 \\
\hline $9.4 \leq \mathrm{NAG} / \mathrm{C}<19.2$ & $-4.0(-4.9,-1.5)$ & $-2.5(-3.1,-0.4)$ & $-8.7(-9.3,-8.0)$ & 0.43 \\
\hline $19.2 \leq \mathrm{NAG} / \mathrm{C}$ & $-1.6(-3.7,-0.1)$ & $-5.1(-7.9,-3.2)$ & -10.5 & 0.060 \\
\hline eGFR $<59$ & $-1.6(-3.4,-0.6)$ & $-2.9(-3.3,-1.1)$ & $-9.3(-10.5,-8.0)$ & 0.56 \\
\hline $59 \leq \mathrm{eGFR}<88$ & $-2.9(-3.8,+2.9)$ & $-5.1(-8.1,-3.4)$ & $+0.2(-3.6,+4.0)$ & 0.022 \\
\hline $88 \leq \mathrm{eGFR}$ & $-4.0(-5.7,-1.5)$ & $-3.4(-4.5,-3.1)$ & $-2.6(-5.0,-0.2)$ & 0.76 \\
\hline
\end{tabular}


(A)

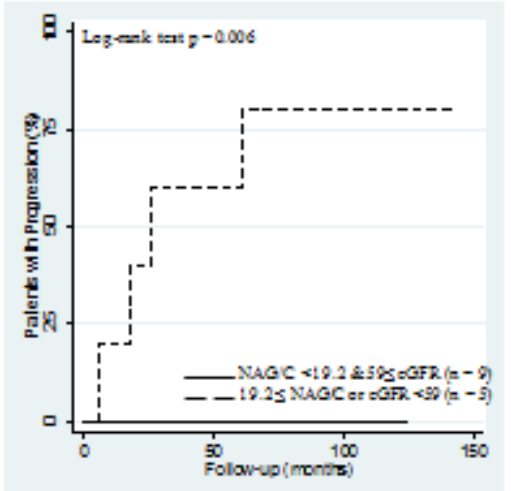

(B)

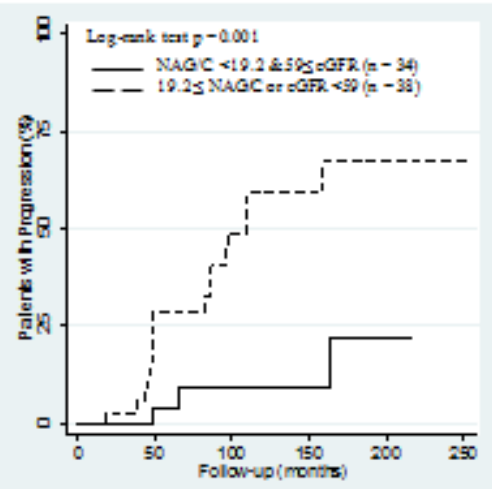

Figure S1. Kaplan-Meier curves for the percentages of subjects with progression with and without nephrotic syndrome $(n=86)$

(A) Subjects without nephrotic syndrome $(n=14)$

(B) Subjects with nephrotic syndrome $(\mathrm{n}=72)$

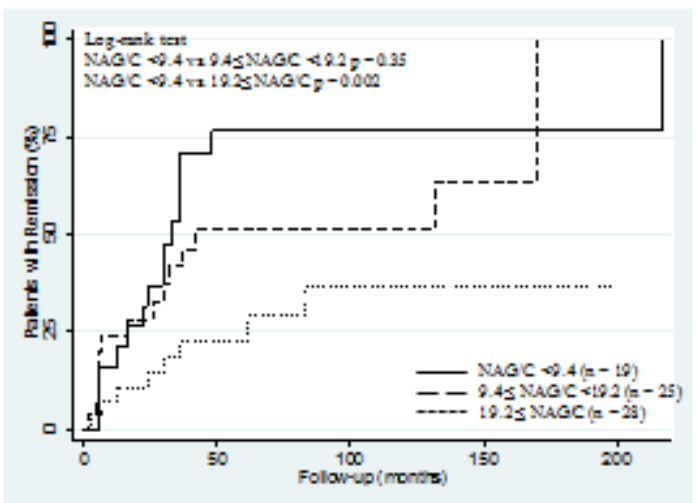

Figure S2. Kaplan-Meier curves for the percentages of nephrotic syndrome subjects with clinical remission by tertiles of $\mathrm{NAG} / \mathrm{C}(\mathrm{n}=72)$ 


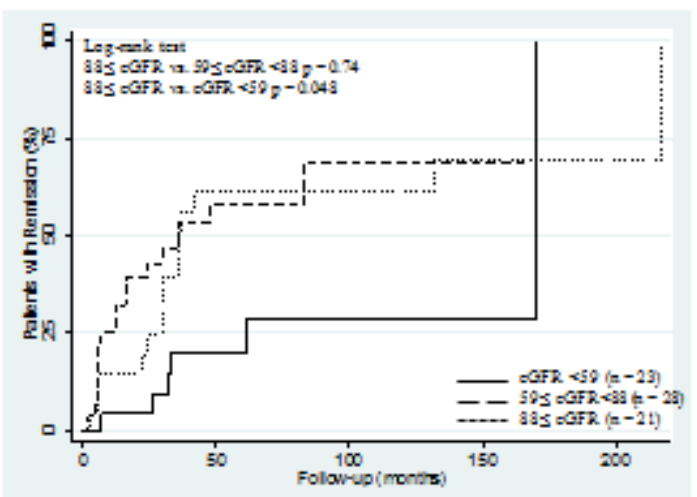

Figure S3. Kaplan-Meier curves for the percentages of nephrotic syndrome subjects with clinical remission by tertiles of eGFR $(n=72)$

(A)

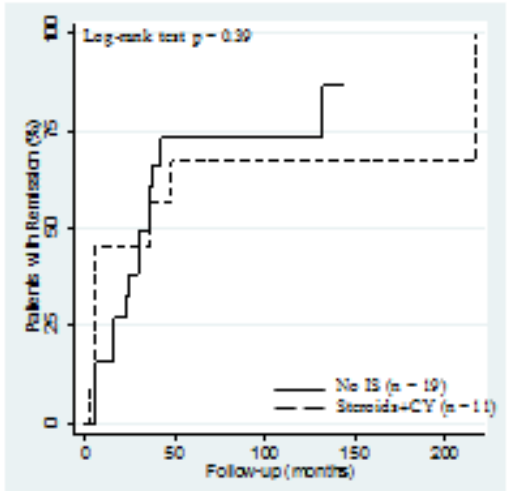

(B)

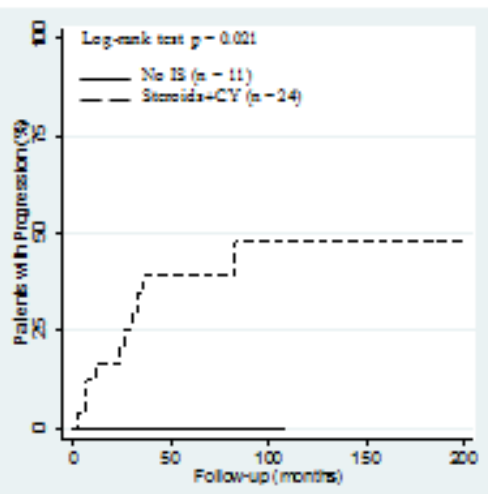

Figure S4. Kaplan-Meier curves for the percentages of subjects with clinical remission with or without immunosuppressive treatment in low and high risk nephrotic syndrome subjects $(\mathrm{n}=65)$

(A) Low risk (NAG/C $<19.2$ and $59 \leq \mathrm{eGFR}$ ) nephrotic syndrome subjects ( $\mathrm{n}=30$ )

(B) High risk $(19.2 \leq \mathrm{NAG} / \mathrm{C}$ or eGFR $<59)$ nephrotic syndrome subjects ( $\mathrm{n}=35$ ) 